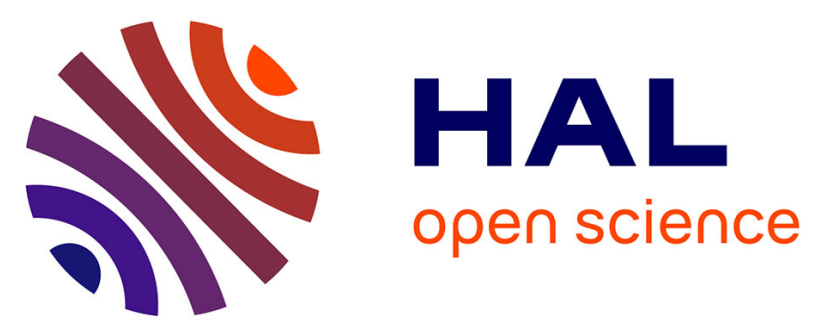

\title{
Design and performance evaluation of an OPC device using a dual-pump polarization-independent FOPA
}

Isaac Sackey, Francesco da Ros, Thomas Richter, Robert Elschner, Mahmoud Jazayerifar, Christian Meuer, Christophe Peucheret, Klaus Petermann, Colja Schubert

\section{To cite this version:}

Isaac Sackey, Francesco da Ros, Thomas Richter, Robert Elschner, Mahmoud Jazayerifar, et al.. Design and performance evaluation of an OPC device using a dual-pump polarization-independent FOPA. 40th European Conference on Optical Communication (ECOC 2014), Sep 2014, Cannes, France. paper Tu.1.4.4, 10.1109/ECOC.2014.6963845 . hal-01069392

\section{HAL Id: hal-01069392 https://hal.science/hal-01069392}

Submitted on 29 Sep 2014

HAL is a multi-disciplinary open access archive for the deposit and dissemination of scientific research documents, whether they are published or not. The documents may come from teaching and research institutions in France or abroad, or from public or private research centers.
L'archive ouverte pluridisciplinaire HAL, est destinée au dépôt et à la diffusion de documents scientifiques de niveau recherche, publiés ou non, émanant des établissements d'enseignement et de recherche français ou étrangers, des laboratoires publics ou privés. 


\title{
Design and Performance Evaluation of an OPC Device Using a Dual-Pump Polarization-Independent FOPA
}

\author{
I. Sackey ${ }^{(1,2)}$, F. Da Ros ${ }^{(2,3)}$, T. Richter ${ }^{(2)}$, R. Elschner ${ }^{(2)}$, M. Jazayerifar ${ }^{(1)}$, C. Meuer ${ }^{(1,2)}$,
} C. Peucheret ${ }^{(4)}$, K. Petermann ${ }^{(1)}$, and C. Schubert ${ }^{(2)}$

(1) Technische Universität Berlin, Fachgebiet Hochfrequenztechnik, Einsteinufer 25, 10587 Berlin,

${ }^{(2)}$ Fraunhofer Institute for Telecommunications, Heinrich Hertz Institute, Einsteinufer 37, 10587 Berlin Germany (Email: isaac.sackey@hhi-extern.fraunhofer.de)

${ }^{(3)}$ Department of Photonics Engineering, Technical University of Denmark, DK-2800 Kgs. Lyngby, DK.

${ }^{(4)}$ FOTON Laboratory, CNRS UMR 6082, ENSSAT, University of Rennes 1, F-22305 Lannion, France.

Abstract. The performance of a polarization-independent fiber-based optical parametric amplifier is experimentally investigated in terms of amplification and wavelength conversion for optical phase conjugation applications using 5×28-GBd PDM 16-QAM signals. Good conjugated signal quality up to $13-d B$ gain is obtained.

\section{Introduction}

The nonlinear Shannon limit represents one of the most critical challenges optical networks will have to address in the near future ${ }^{1}$. In this context, a renewed interest for nonlinear compensation has led to several demonstrations both in the digital and the optical domains ${ }^{2-4}$. Optical phase conjugation (OPC) is a well-known technique allowing inverting the signal spectrum, therefore enabling the compensation of dispersion and Kerr effect accumulated through propagation by further transmission of the conjugated signal. For practical application, an OPC device should provide broadband operation as well as modulationformat transparency and polarization independence. Towards this aim, nonlinear compensation was demonstrated in Ref. 5 for 16-quadrature amplitude modulation (QAM) signals. However, operation was restricted to single polarization and limited by the penalty introduced by the OPC itself. In Ref. 2, polarization-independence was achieved, but only quadrature phase-shift keying (QPSK) operation was reported.

In this work, we investigate the potential of using a broadband dual-pump fiber optical parametric amplifier (FOPA) to provide polarization-independent and low penalty OPC opera- tion for a 5-channel wavelength-division multiplexed (WDM) 28-GBd polarization division multiplexed (PDM) 16-QAM signal. The relative performances of the amplified signal and idler are evaluated in terms of polarization sensitivity and bit-error ratio (BER), highlighting the key optimization aspects for the successful design of a black-box OPC device.

\section{Experimental setup}

The experimental setup is presented in Fig. 1. The setup consists of a 5-channel 28-GBd PDM 16-QAM transmitter, the FOPA, and a coherent receiver. Five continuous wave (cw) external cavity lasers (ECLs) on a $50-\mathrm{GHz}$ grid centered at $1550.12 \mathrm{~nm}$ were modulated with 16-QAM at $28 \mathrm{GBd}$ using an IQ modulator driven by two channels of a 56-GS/s arbitrary waveform generator (AWG). A polarization emulator (PolMux) was then used to generate a PDM signal, followed by a decorrelation stage to fully decorrelate all five channels ${ }^{6}$. Before entering the FOPA, a scrambler was used to randomize the state-of-polarization (SOP) of the signal.

The FOPA consists of two ECLs serving as cw pump sources at $1534 \mathrm{~nm}$ and $1574 \mathrm{~nm}$ with linewidths of $25 \mathrm{kHz}$ and $100 \mathrm{kHz}$, respectively. The pumps were separately modulated using phase modulators (PMs) driven in a coun-

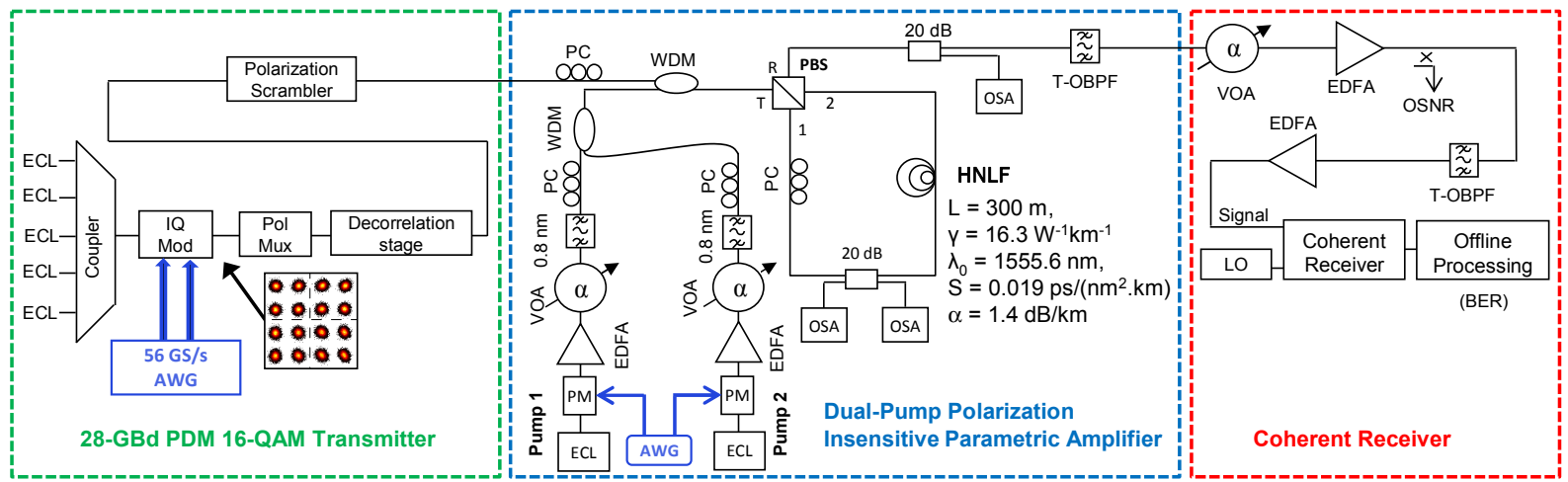

Fig. 1: Experimental setup. 
ter-phasing fashion by two sinusoidal tones (69 $\mathrm{MHz}$ and $253 \mathrm{MHz}$; modulation index of 1.4 radians) generated by another two-channel AWG in order to increase the stimulated Brillouin scattering (SBS) threshold and to minimize the transfer of pump phase modulation to the generated idlers ${ }^{7}$. The pumps were amplified by EDFAs (output optical signal-tonoise ratio (OSNR) of $59 \mathrm{~dB}$ and $56 \mathrm{~dB}$ for the C- and L-band pumps, respectively) and combined with a WDM coupler. The pump power levels were controlled via variable optical attenuators (VOAs) thus ensuring constant pumps OSNR values. Optical band-pass filters (OBPFs) with a full-width at half-maximum (FWHM) bandwidth of $0.8 \mathrm{~nm}$ suppress the outof-band amplified spontaneous emission (ASE). Pumps and signal were combined using a WDM coupler before being sent to the diversity loop consisting of a polarization beam splitter (PBS) and a piece of highly nonlinear fiber (HNLF) (length $300 \mathrm{~m}$, zero dispersion wavelength $1555.6 \mathrm{~nm}$, nonlinear coefficient $16.3 / \mathrm{W} / \mathrm{km}$, attenuation $1.4 \mathrm{~dB} / \mathrm{km}$, dispersion slope $0.019 \mathrm{ps} / \mathrm{nm}^{2} / \mathrm{km}$ ). The total signal power at the PBS was kept constant at $-5 \mathrm{dBm}$ throughout the evaluation except for the saturation analysis. In order to match the gain in both loop directions, the SOP of the pumps was tuned via two polarization controllers (PCs) and the SOP of the signal was randomized using the polarization scrambler. A 20-dB coupler in the loop was used to monitor the pumps power levels in both directions of the loop. A PC in the loop was used to ensure that the waves at the output of the HNLF exit at port-R of the PBS. The output spectra after the diversity loop were monitored by an optical spectrum analyzer (OSA) via a 20-dB coupler. At the FOPA output a tunable (T) OBPF with 4-nm FWHM bandwidth was used to select either the amplified signal or the idler bands and to suppress the pumps.

At the receiver, the signal was noise-loaded, the channel under test was selected by a T-OBPF and injected together with a local oscillator (LO) with $100-\mathrm{kHz}$ linewidth in a coherent receiver consisting of a $90^{\circ}$ optical hybrid, balanced photodiodes (BPD) and a $40 \mathrm{GS} / \mathrm{s}$ real-time sampling scope (RTO). Offline processing was then performed, including frequency-offset estimation, adaptive equalization, carrier phase estimation and error counting ${ }^{6}$.

\section{Results and Discussions}

The transfer of pump phase noise to the idler strongly degrades the quality of the idler. Therefore the counter-phasing was optimized using a single channel $\mathrm{cw}$ signal at $1550.12 \mathrm{~nm}$ injected into the FOPA. The generated idler was detected using the RTO and its spectrum was monitored to assess the amount of phase modulation at $69 \mathrm{MHz}$ and $253 \mathrm{MHz}$ being transferred from the pumps. As shown in Fig. $2 a$, regardless of the On-Off gain values $(5 \mathrm{~dB}, 10 \mathrm{~dB}$ and $13 \mathrm{~dB}$ were considered in this investigation), the suppression ratio between the carrier and the two tones and their harmonics was higher than $33 \mathrm{~dB}$, showing that low phase modulation was transferred to the idler ${ }^{7}$.

Fig. 2b shows the On-Off gain and conversion efficiency (CE) as functions of the signal power at the PBS input. The CE is defined as the ratio of idler to signal powers with pumps off measured at the FOPA output. This particular characterization was done in a polarization dependent configuration. The polarizations of the pumps and a cw signal at $1550.12 \mathrm{~nm}$ were aligned to propagate in one direction of the diversity loop without polarization scrambling. As expected, the difference between gain and CE decreases with increasing the gain. Higher gains begin to saturate at lower input powers than lower gain levels. However, gain reduction of $1 \mathrm{~dB}$ is obtained at a signal input power of $+5 \mathrm{dBm}$ even when the gain is $13 \mathrm{~dB}$.

For the gain bandwidth measurement, the WDM coupler (signal-pump combiner) was temporarily replaced with a $10-\mathrm{dB}$ coupler. This characterization was performed with the pumps SOPs set for polarization-independent operation, and polarization-scrambling of the cw-probe signal, whose wavelength was swept from $1535 \mathrm{~nm}$ to $1572 \mathrm{~nm}$. The total pump power into the loop was set to $28.2 \mathrm{dBm}$, $29.9 \mathrm{dBm}$, and $30.8 \mathrm{dBm}$ for $5 \mathrm{~dB}, 10 \mathrm{~dB}$, and $13 \mathrm{~dB}$ On-Off gain, respectively. The results are summarized in Fig. $2 \mathrm{C}$ and show a flat gain with $1-\mathrm{dB}$ bandwidth of $24 \mathrm{~nm}$ at $5-\mathrm{dB}$ On-Off gain which reduces to $18 \mathrm{~nm}$ at $13 \mathrm{~dB}$. A slight gain tilt resulting from the Raman effect is also visible though its impact was kept low by setting the C-band pump power $\sim 1.8 \mathrm{~dB}$ higher than the L-band pump power. Fig. 2c also illustrates the PDG for the modulated signal and its idler measured using the zero-span function of the OSA while scrambling a single-channel 28-GBd 16-QAM signal with the PDM emulator bypassed. Averaging effects in the PDG measurement are minimized by the use of a low scrambling rate. The signal wavelength varied from $1542.5 \mathrm{~nm}$ to $1565 \mathrm{~nm}$, i.e. the flat region of the gain profile. The measurement was done for $5-\mathrm{dB}, 10-\mathrm{dB}$, and $13-\mathrm{dB}$ gain showing low $P D G$ values ranging from $0.2 \mathrm{~dB}$ to $0.5 \mathrm{~dB}$ with similar signal/idler values. Note that at higher gain, the pump-power splitting is more critical 
(a)

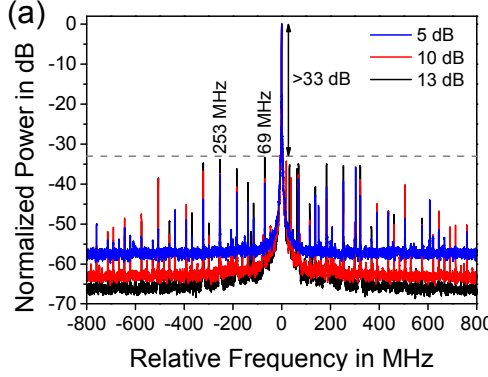

(d)

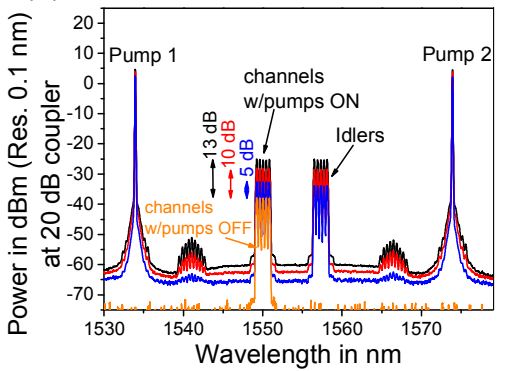

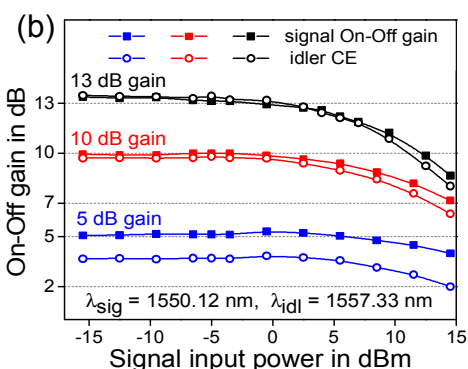

(e)

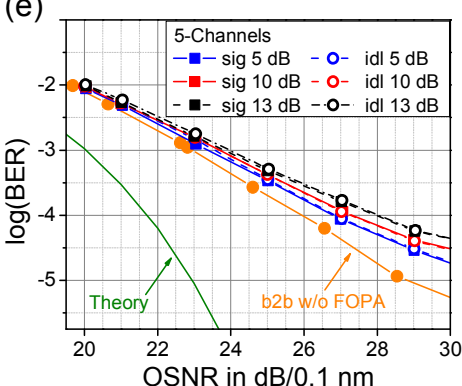

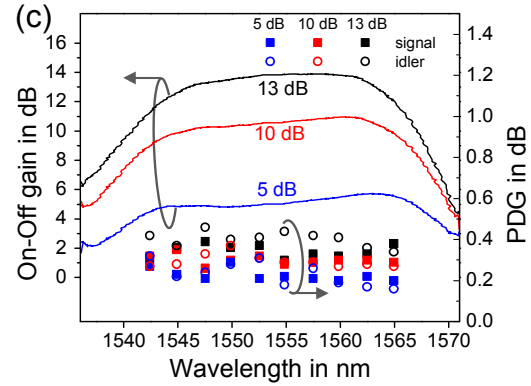

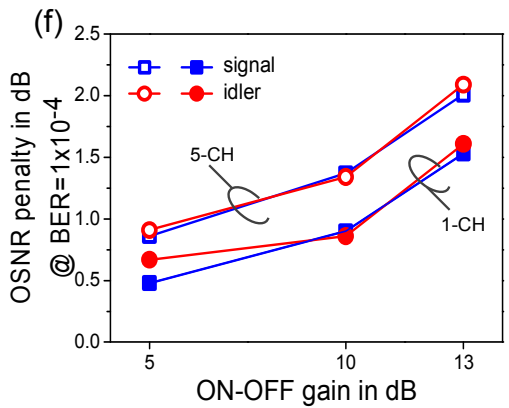

Fig. 2: (a) Spectra of RTO-acquisition showing suppression of pump phase modulation tones on the generated idler. (b) Gain saturation characteristics. (c) Gain bandwidth and PDG for the amplified signals and the idlers. (d) Spectra at the HNLF output for the different gain levels showing the 5-channel DWDM signal and the generated idlers. (e) 5-channel BER curves for signal and idler at different gain levels. (f) OSNR penalties at a BER of $1 \times 10^{-4}$ for both signal and idler as functions of On-Off gain.

due to the exponential gain regime, resulting in slightly higher PDG values.

Fig. 2d shows the WDM spectra after the diversity loop with pumps On and Off for 5-dB, 10-dB, and 13-dB gain with a total WDM-signal input power into the loop of $-5 \mathrm{dBm}$. This power was used to operate the FOPA in the linear regime $^{8}$. The wavelengths of the WDM channels have been selected to be within the flat range of the gain spectra. The BER performance for the amplified signals and the generated idlers were measured for the 5-channel WDM input as well as for a single-channel input (WDM centerchannel). The WDM results are shown in Fig. 2e in which the average BER of the five channels is plotted as a function of the OSNR (in $0.1 \mathrm{~nm}$ reference bandwidth). Very small BER differences were identified between the channels. A summary of the measured OSNR penalties for both single and WDM scenarios at a BER of $1 \times 10^{-4}$ is depicted in Fig. $2 f$ for the selected On-Off gain values. Signal and idler show similar BER performances, demonstrating the effectiveness of the scheme for OPC operation. However, the distortion experienced in the FOPA increases with gain and is stronger in the WDM case, indicating that a limited gain of $5 \mathrm{~dB}$ would be more beneficial for practical implementation.

The degradation is attributed to distortions such as self phase modulation in the case of the single-channel and cross phase modulation, cross gain modulation, and inter-channel four-wave mixing in the case of $\mathrm{WDM}^{8}$. Additionally, the diversity loop operation introduces extra penalty compared to a polarization dependent scheme ${ }^{8}$ limiting the gain. Further studies are therefore required.

\section{Conclusion}

A polarization-independent FOPA for OPC was experimentally demonstrated at $5 \mathrm{~dB}, 10 \mathrm{~dB}$, and $13 \mathrm{~dB}$ On-Off gain for 28-GBd PDM 16-QAM signals in a 5-channel WDM system. Signal and idler investigations showed OSNR penalties below $1 \mathrm{~dB}$ for both single channel and a 5-channel WDM cases at 5-dB On-Off gain and at a BER of $1 \times 10^{-4}$. The maximum polarization dependent gain was also measured to be below $0.5 \mathrm{~dB}$ across a 22.5-nm wavelength range $(1542.5 \mathrm{~nm}$ to $1565 \mathrm{~nm})$. The scheme is well suited for in-line OPC applications at 5-dB gain for gain-transparent operation with minimal penalty from the FOPA.

\section{Acknowledgements}

We thank the German Research Council (DFG) under projects GR 3774/1-1 and PE 319/26-1. We also thank the Danish Research Council for Technology and Production Sciences under project 09-066562.

\section{References}

[1] A. D. Ellis, et al., "Approaching the non-linear Shannon limit", JLT., Vol. 28, p. 423 (2010)

[2] D. Rafique, et al., "Digital back-propagation for spectrally efficient WDM $112 \mathrm{Gbit} / \mathrm{s}$ PM m-ary QAM transmission", Opt. Express, Vol. 19, p. 5219 (2011)

[3] $\mathrm{H}$. Hu, et al., "Fiber nonlinearity compensation of an 8-channel WDM PDM-QPSK signal using multiple phase conjugations", Proc. OFC 2014, paper M3C.2.

[4] X. Liu, et al., "Phase-conjugated twin waves for communication beyond the Kerr nonlinearity limit", Nat.Phot. Vol.7, p.560 (2013).

[5] L. B. Du, et al, "Fiber nonlinearity compensation for OFDM super-channels using optical phase conjugation", Opt. Express, Vol 20, p. 19921 (2012).

[6] F. Da Ros et al., "Kerr Nonlinearity Compensation in a $5 \times 28-\mathrm{GBd}$ PDM 16-QAM WDM System Using Fiber-Based Optical Phase Conjugation", ECOC 2014, submitted.

[7] T. Richter et al., "Parametric amplification of $112 \mathrm{Gbit} / \mathrm{s}$ polarization multiplexed DQPSK signals in a fiber loop configuration," IEEE JSTQE Vol. 18, p. 988, (2012).

[8] I. Sackey et al.,"Characterization of a FOPA in a $5 \times 28-G B d$ 16-QAM DWDM system", Proc. OFC 2014, paper W3E.3. 